LA-UR-99-3276

Approved for public release; distribution is unlimited.

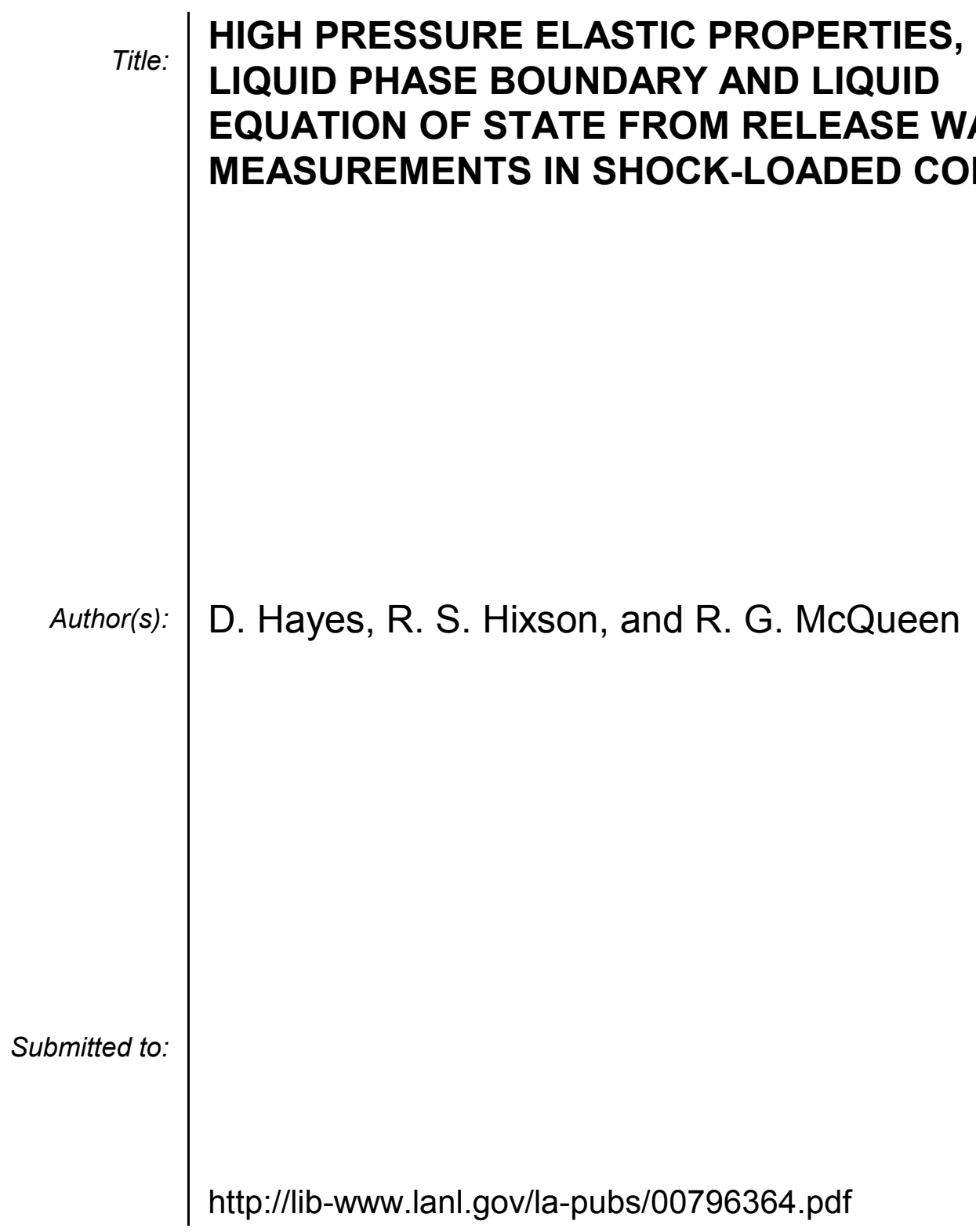

Los Alamos National Laboratory, an affirmative action/equal opportunity employer, is operated by the University of California for the U.S. Department of Energy under contract W-7405-ENG-36. By acceptance of this article, the publisher recognizes that the U.S. Government retains a nonexclusive, royaltyfree license to publish or reproduce the published form of this contribution, or to allow others to do so, for U.S. Government purposes. Los Alamos National Laboratory requests that the publisher identify this article as work performed under the auspices of the U.S. Department of Energy. Los Alamos National Laboratory strongly supports academic freedom and a researcher's right to publish; as an institution, however, the Laboratory does not endorse the viewpoint of a publication or guarantee its technical correctness. 


\title{
HIGH PRESSURE ELASTIC PROPERTIES, SOLID-LIQUID PHASE BOUNDARY AND LIQUID EQUATION OF STATE FROM RELEASE WAVE MEASUREMENTS IN SHOCK-LOADED COPPER *
}

\author{
D. Hayes, R. S. Hixson, and R. G. McQueen ${ }^{\dagger}$ \\ Los Alamos National Laboratory, Los Alamos, New Mexico 87545 USA
}

\begin{abstract}
Measured release wave speeds in shock-loaded copper give information on high-pressure elastic properties up to the solid-liquid phase boundary at $2.32 \pm 0.05 \mathrm{Mbar}$. For impact pressures above $2.65 \pm 0.06 \mathrm{Mbar}$, the shocked state is completely melted copper and those release wave speeds give new experimental information on the liquid equation of state. The Hugoniot derived from solid and liquid equations of state developed from these experiments and the measured location of the phase boundary compare favorably with existing $a b$ initio calculations.
\end{abstract}

\section{INTRODUCTION AND SUMMARY}

Copper is among the most studied materials using shock-compression techniques. Numerous investigators have performed experiments on copper applying shock stresses ranging from a few kilobars to over nine megabars.(1,2) The resulting Hugoniot measurements give accurate information on the equation of state at high pressure and temperature. Several researchers also measured sound speed in the shocked state.(3-5) In this work we report additional measurements (using the overtaking-wave technique(6) to measure the sound speeds) and have analyzed all available data to obtain: elastic properties of the solid at large compression; equation of state of the liquid; and the location of the solid-liquid phase boundary.

Figure 1 is a summary of all measured Hugoniot and sound speed data and also includes calculated bulk sound speeds $c_{B}$ for the solid and liquid, which are described later.

\footnotetext{
* This work supported by the US Department of Energy.

† Some of the experiments reported here were performed by shock-wave pioneer Bob McQueen in the 1980's. Bob passed away before his study was completed.
}

For shocks below 2.32 Mbar, the material remains in the solid phase. The measured sound speed is therefore the longitudinal elastic wave velocity $c_{L}$. A complete equation of state for the solid allows calculation of the bulk sound speed $c_{B}$. By assuming copper is isotropic, $c_{L}$ and $c_{B}$ are sufficient to calculate other elastic properties. Results show the shear modulus $G$ rises gradually along the Hugoniot and then drops precipitously as the shocked state nears the phase boundary. The Hugoniot stress at which the measured shear modulus vanishes is assumed to indicate the intersection of the Hugoniot and the solidliquid phase boundary.

Analysis of sound speed and Hugoniot data above $2.65 \mathrm{Mbar}$ show that Grüneisen's ratio $\gamma$ of shockmelted copper is constant (1.55). The equation of state for liquid copper developed below will assume constant $\gamma$. Shock pressures between 2.32 and $2.65 \mathrm{Mbar}$ therefore produce partially melted copper. The location and traverse of the Hugoniot along the phase boundary are in good agreement with ab initio 
Los Alamos National Laboratory, an affirmative action/equal opportunity employer, is operated by the University of California for the U.S. Department of Energy under contract W-7405-ENG-36. By acceptance of this article, the publisher recognizes that the U.S. Government retains a nonexclusive, royaltyfree license to publish or reproduce the published form of this contribution, or to allow others to do so, for U.S. Government purposes. Los Alamos National Laboratory requests that the publisher identify this article as work performed under the auspices of the U.S. Department of Energy. Los Alamos National Laboratory strongly supports academic freedom and a researcher's right to publish; as an institution, however, the Laboratory does not endorse the viewpoint of a publication or guarantee its technical correctness. 


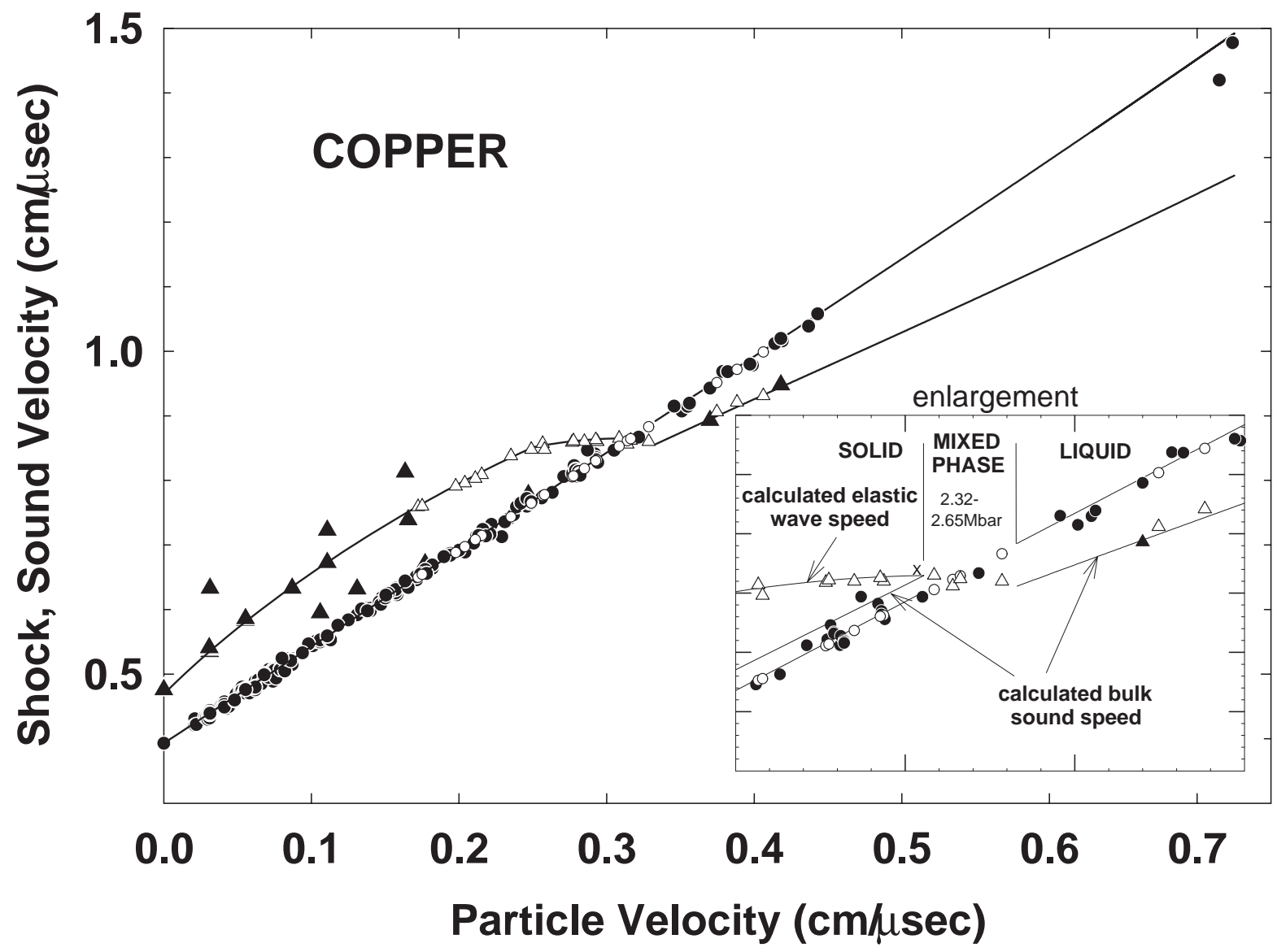

FIGURE 1. Summary of shock and sound speed measurements in copper. Hugoniot (circles); Sound speed (triangles). The open symbols are data reported here for the first time. The three solid triangles which fall well above the solid line were obtained from thin specimens and on the advice of the experimenter have been dropped from all subsequent analysis. There are several solid triangles which fall well below the solid line including several which are masked by Hugoniot data. These were obtained forty years ago by the best available method but are also suspect. They have also been excluded from consideration in this work. All solid lines are calculated. The central portion of this figure is enlarged in the insert. The lower left hand corner of this insert has coordinates $(0.25,0.7)$; major divisions are $0.05 \mathrm{~cm} / \mu \mathrm{s}$.

Preliminary results on stainless steel-316, which will be reported later, are qualitatively the same as those for copper. However more sparse data mean that uncertainties in quantitative results are larger.

\section{SOLID}

The Mie-Grüneisen equation of state which exactly produces a linear shock velocity-particle velocity Hugoniot, $u_{s}=c_{0}+s u_{p}$, is

$$
P=\frac{\rho_{0} c_{0}^{2} \epsilon}{(1-s \epsilon)^{2}}\left(1-\frac{\rho \gamma}{2}\left(V_{0}-V\right)\right)+\rho \gamma E
$$

where $\rho, E, \epsilon$, and $V$ are density, specific internal energy, engineering strain and specific volume, respectively. The product $\rho \gamma$ is commonly assumed constant and we use the value given by McQueen et al.(8) Equation (1), along with an assumed constant specific heat at constant volume, is thermodynamically complete allowing subsequent estimates of Hugoniot temperature and entropy. The origin of energy and entropy were selected as zero in the solid at ambient conditions. All numerical constants used in 
TABLE 1.

Solid Equation of State Constants

$\rho_{0}=8.93\left(\mathrm{~g} / \mathrm{cm}^{3}\right)$

$c_{0}=0.3928(\mathrm{~cm} / \mu \mathrm{s})$

$s=1.489$

$\rho \gamma=8.93 \times 1.96\left(\mathrm{~g} / \mathrm{cm}^{3}\right)$

$C_{V}=3 R=4 \times 10^{-6}\left(\mathrm{~cm}^{2} / \mu \mathrm{s}^{2} \mathrm{~K}\right)^{a}$

$T_{0}=293 \mathrm{~K}$

$\nu=\max (0.356+P / 21.16,0.26+P / 9.667)$,

and constrained: $-1<\nu<0.5,(P$ in Mbar $)$.

Liquid Equation of State Constants

$\rho_{0}=8.00\left(\mathrm{~g} / \mathrm{cm}^{3}\right)$

$\gamma=1.55$

$C_{V}=C_{V}$ (solid)

$T_{0}=1354 \mathrm{~K}$

Along $T=T_{0}, K_{T}=0.7743(1+5.409 \epsilon$

$\left.+3.548 \epsilon^{2}+85.451 \epsilon^{3}\right)(\mathrm{Mbar})^{b}$

Phase Boundary at Zero Pressure

$T_{\text {melt }}=1354 \mathrm{~K}$

$\Delta H_{\text {fusion }}=2.055 \times 10^{-3}\left(\mathrm{~cm}^{2} / \mu \mathrm{s}^{2}\right)$

${ }^{a}$ Note: $C_{V}$ is expressed per gram and not per mole.

${ }^{b}$ For a constant $\gamma, C_{V}$ equation of state the general expression for the isothermal bulk modulus must be of the form $K_{T}=$ $K_{T}\left(V, T_{0}\right)+\left(\gamma C_{V} / V\right)\left(T-T_{0}\right)$ to insure existence of a unique Helmholtz potential.

this study are in Table 1.

Bulk sound speed on the Hugoniot derives from constant entropy differentiation of Eq. (1), evaluated on the Hugoniot.

$$
\frac{c_{B}^{2}}{V^{2}}=\left(1-\frac{\gamma \epsilon V_{0}}{2 V}\right)\left(-\frac{d P}{d V}\right)_{H}+\frac{\rho \gamma}{2} P_{H} .
$$

Elastic properties are expected to be functions of both temperature and density. However, data are only available along a single path, the Hugoniot. Poisson's ratio $\nu$ for each shock datum, calculated from

$$
\nu=\left(3 c_{B}^{2}-c_{L}^{2}\right) /\left(3 c_{B}^{2}+c_{L}^{2}\right)
$$

is plotted against Hugoniot pressure, a convenient parameter. (See Fig. 2.) A pair of straight line segments fit to these data and Eq. (2) for the bulk sound speed are used in all subsequent calculations of elastic properties.

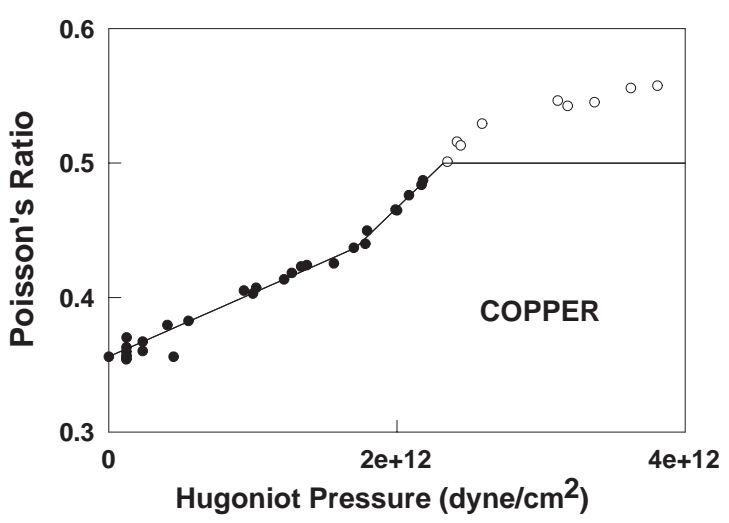

FIGURE 2. Experimental Poisson's ratio plotted against Hugoniot Pressure is fit with straight-line segments. Experiments with shock pressure exceeding 2.32 Mbar lead to non-physical values of Poisson's ratio greater than 0.5 . These are assumed to be mixedphase or liquid and thus the analysis does not apply. They have been included in the figure as open symbols. They have been included for information only and are not part of the data set for the solid.

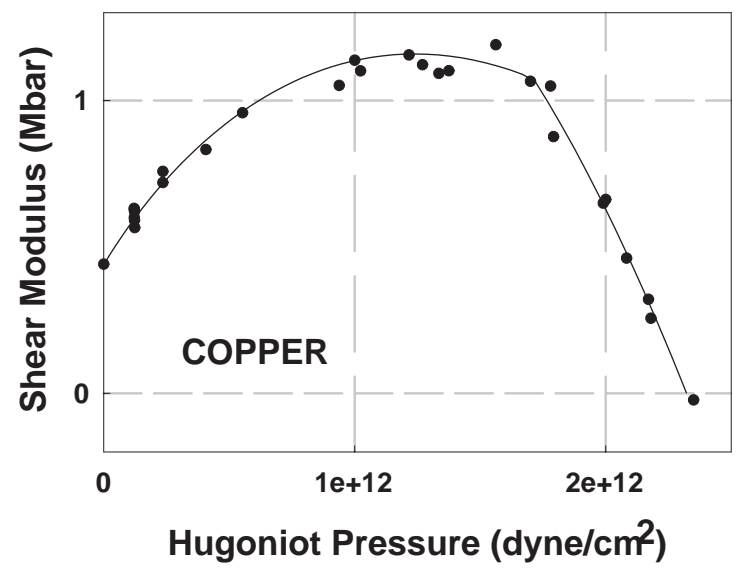

FIGURE 3. Measured Shear Modulus along the Hugoniot.

Poisson's ratio is seen to rise with shock pressure from its zero-pressure value of 0.356 approaching the value for a liquid of 0.5 at 2.32 Mbar. Interpretation of this behavior is clearer in Fig. 3, which shows the shear modulus rising from its ambient value of $441 \mathrm{kbar}$ to over $1189 \mathrm{kbar}$ and dropping precipitously as the shock strength approaches the value at melt. The point at which the shear modulus vanishes is assumed incipient melt. 
Point " $\mathrm{X}$ " in the inset box of Fig. 1 re-displays the vanishing shear modulus as a coincidence of the longitudinal sound speed with the bulk sound speed. The agreement of this pressure with calculated incipient melt(7) shown in figure 6 leads us to conclude that onset of melt is sufficient to cause loss of the ability of copper to support shear stress.

\section{LIQUID}

Grüneisen's ratio for shocked liquid copper is calculated from the Hugoniot energy jump condition which has been expressed

$$
\frac{\gamma \epsilon V_{0}}{2 V}=\frac{\left(\frac{d P}{d V}\right)_{H}-\left(\frac{\partial P}{\partial V}\right)_{S}}{\left(\frac{d P}{d V}\right)_{H}+\frac{P}{\epsilon V_{0}}} .
$$

Measurement of the shock state $(P, V, \epsilon)$, sound speed $\left(\sqrt{(\partial P / \partial \rho)_{S}}\right)$ and the slope of the Hugoniot allow calculation of the Grüneisen ratio for each shocked copper datum in the liquid state. For the slope of the Hugoniot we use the calculated linear shock-velocity, particle-velocity of McQueen $e t$ $a l .{ }^{*}(8)$ Figure 4 shows that for shock pressure above 2.65 Mbar, the measured values lie in the narrow band $\gamma=1.55 \pm 0.05$, which is the estimated precision. Overall accuracy is estimated at $10 \%$ owing to the sensitivity of the calculations to the slope of the Hugoniot. Further experimentation which better defines the slope of the Hugoniot can be used to improve the accuracy of these measured Grüneisen ratios.

The complete equation of state for liquid copper is constructed by assuming Grüneisen's ratio and constant volume specific heat are each constant ${ }^{\dagger}$ and the ambient temperature isothermal bulk modulus is a cubic polynomial in strain. A generalized regression finds the best values for polynomial coefficients

\footnotetext{
* The only use of the liquid linear fit is to evaluate $(d P / d V)_{H}$ in Eq. (4). The $\gamma$ 's determined in this way are seen to be constant. All subsequent calculations are made with a constant $\gamma, C_{V}$ equation of state which has the reference isotherm given in Table 1.

$\dagger$ This is known to be an approximation. We ignore, for instance, the electronic specific heat, which will make our temperature calculation slightly high.
}

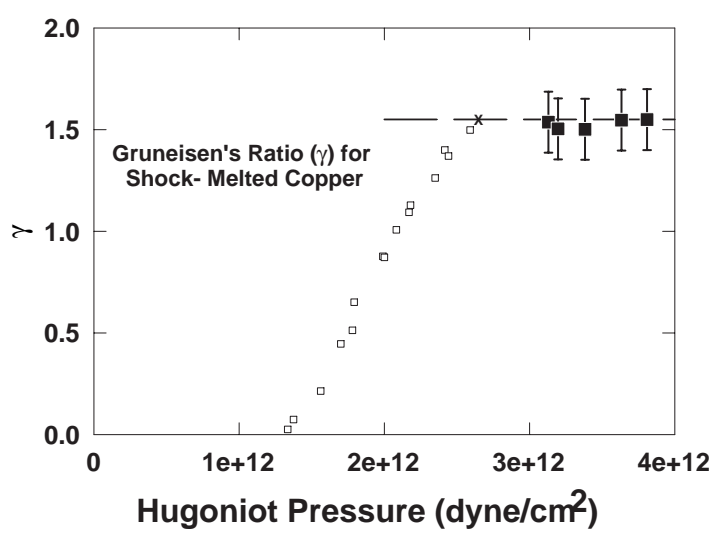

FIGURE 4. Measured Grüneisen's ratio for Liquid Copper on the Hugoniot. The open circles are assumed not totally melted. The exit of the Hugoniot from the mixed phase region is denoted "X". The open symbols are included for information only and are not part of the data set for liquid copper.

which make the complete equation of state simultaneously fit the following available data: the Hugoniot data, sound speeds in the shocked state, zero pressure density and coefficient of expansion, sound speed and its temperature derivative in zero pressure liquid copper. All data are fit to within experimental error which is typically better than $2 \%$. The Hugoniot and sound speed for liquid copper calculated with the resulting complete equation of state are shown in Fig. 1. Zero pressure sound speed measurements $(9)$ along with the calculated values are given in Fig. 5 .

\section{MIXED PHASE}

The measured entrance of the Hugoniot into the mixed phase region is taken as the pressure at which Poisson's ratio first equals 0.5. Error is taken from graphical uncertainty in this intersection. This point is $2.32 \pm 0.05 \mathrm{Mbar}$. The measured exit of the Hugoniot from the mixed-phase region is the point denoted " $X$ " in Fig. 4. Error was estimated in the same way. The exit is $2.65 \pm 0.06 \mathrm{Mbar}$. These agree well with the ab initio calculations of Moriarty.(7) (See Fig. 6.) The Hugoniot of the solid from our complete equation of state agrees well also. However 


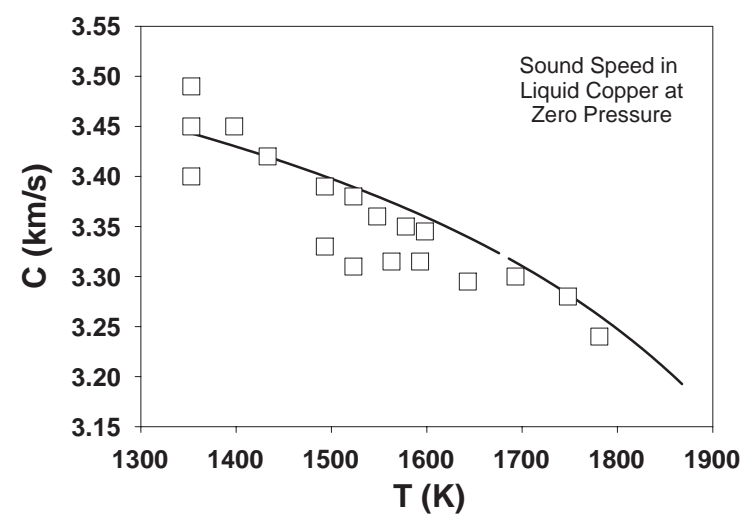

FIGURE 5. Measured sound speed in liquid copper at zero pressure. The solid curve is calculated from our complete equation of state.

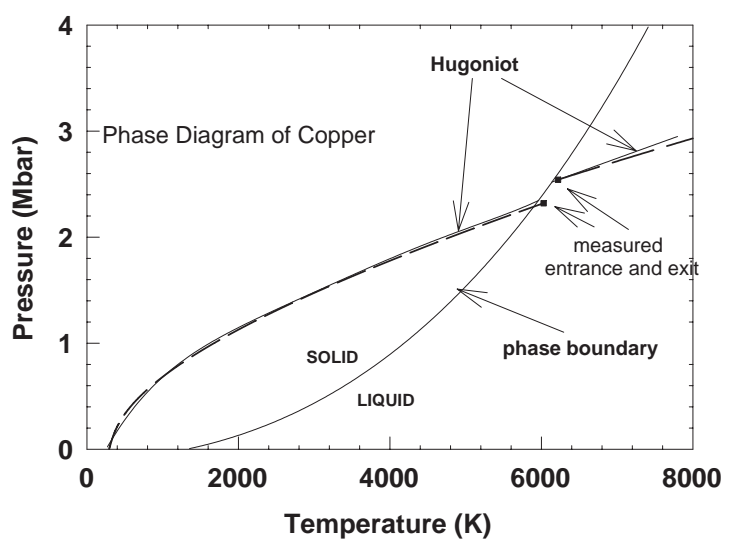

FIGURE 6. Measured entrance and exit of the Hugoniot from the mixed phase of copper compared with Moriarty (solid) and compared with this work (dashed and data).

there is small disagreement between our calculation and Moriarty's for the Hugoniot of the liquid. Our method does a poor job of estimating the location of the phase boundary and we have not included it in the figure.

\section{DISCUSSION}

Determination of the location of the copper solidliquid phase boundary solely from shock speed measurements is difficult.(10) The density of the liquid phase is smaller than that of the solid so that increased compression at higher Hugoniot pressures requires that the Hugoniot "traverse" the mixedphase region over a large pressure range. Furthermore, unlike most solid-solid transformations, melting produces no two-wave structure and the shock/particle velocity Hugoniot curve displays only a very small change in slope. Thus the problem reduces to finding the intersection of two nearly parallel, slightly curved lines that inherently produces large uncertainties. In this work we have attempted to locate the phase boundary experimentally using a different approach. Because of simultaneous shock and sound speed measurements in the shocked state it is possible to find the entrance of the Hugoniot into the mixed phase region where the shear modulus vanishes. Similarly, the exit of the Hugoniot from the mixed phase region is supposed at the pressure above which the Grüneisen ratio is constant. These entrance and exit points agree well with existing $a b$ initio calculations validating the assumptions.

One distinctive feature of the copper sound velocity data gives insight into the shock-induced melting process. Several bcc metals have been studied using the release overtake method and those data show a large drop in sound speed with small increases in pressure (over about $40 \mathrm{kbar}$ ) which was interpreted to indicate the onset of melting. This change in sound velocity was consistent with transition from solid to liquid, i.e. longitudinal to bulk sound speed.(11) These studies acknowledged the possibility the drop in sound speed may not exactly coincide with at phase boundary. It is clear from Fig. 7 that no rapid drop in sound speed with pressure is observed in copper, an fcc metal. Instead, a $500 \mathrm{kbar}$ wide plateau is observed. The analysis presented here gives a possible explanation for this behavior. Copper shows a gradual decrease in shear modulus as the phase boundary is approached. This drop is more gradual than that observed in bcc metals studied to date.

Other explanations may exist for our data on the 


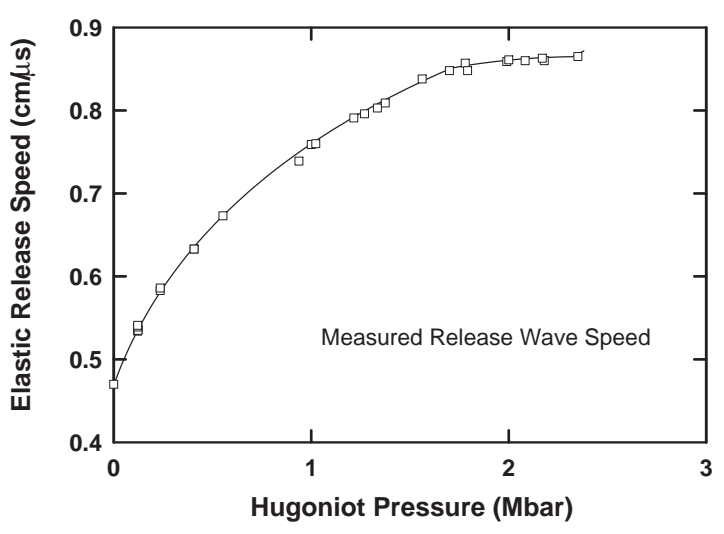

FIGURE 7. Longitudinal Sound Speed Along the Hugoniot.

solid. ${ }^{\ddagger}$ Chemical segregation and higher defect concentration adjacent to grains can result in substantial local free energy changes.(12-14) Strain-induced premelting along grain boundaries with profuse dislocations has been documented in copper bicrystals at temperatures as low as 70 percent of the melt temperature. Shocks also interact with these heterogeneities, pores or inclusions that can lead to differential heating immediately behind the shock. It is possible that the gradual drop in the shear modulus reflects the influence of non-equilibrium melting. Future soft recovery experiments will quench lower melting-point metals, such as $\mathrm{Pb}, \mathrm{Bi}$, and $\mathrm{Sn}$ to investigate this phenomenon. Recovery of partially melted copper is problematic.

It did not escape the authors' attention that Poisson's ratio does not smoothly traverse the region 0 2.32 Mbar. (See Fig. 2) There is a distinct kink in the data at about $1.85 \mathrm{Mbar}$ that we fit with two straightline segments. We have no explanation for this behavior and do not know if it relates to the area of focus of this work.

\section{CONCLUSIONS}

Simultaneous measurement of Hugoniot state and sound speed offers new information on elastic prop-

\footnotetext{
$\mp$ We are indebted to G.T. Gray III for the following observations.
}

erties at high pressure for solid copper and leads to formulation of a complete equation of state for liquid copper. Copper is observed to lose its shear modulus upon incipient melt. Grüneisen's ratio for the liquid is observed constant. The measured intersection of the Hugoniot with the phase boundary agrees well with published calculations.

\section{REFERENCES}

1. M. van Thiel, Compendium of shock wave data, UCRL 50108 rev. 1, vol. 1, Technical report, Lawrence Livermore National Laboratory, 1981.

2. A. G. Mitchell and W. J. Nellis, J. Appl. Phys. 52, 3363 (1981).

3. L. V. Al'tshuler, L. V. Kormer, M. I. Brazhnik, L. Vladimirov, and A. I. Funtikov, Sov. Phys.-JETP 2, 766 (1960).

4. L. C. Chhabildas, Time resolved wave profile measurements in copper to megabar pressures, in High Pressure in Research and Industry; 8th AIRAPT Conference, edited by T. J. C. M. Backman and L. Tegner, pages 183-189, University of Upsula, Sweden, 1981.

5. L. Chhabildas-private communication.

6. R. G. McQueen, J. W. Hopson, and J. N. Fritz, Rev. Scient. Instr. 53, 245 (1982).

7. J. A. Moriarty, High pressure ion-thermal potentials of metals from $a b$ initio interatomic potentials, in Shock Waves in Condensed Matter, edited by Y. M. Gupta, pages 101-106, Plenum Publishing Company, 1986.

8. R. G. McQueen, S. P. Marsh, J. W. Taylor, J. N. Fritz, and W. J. Carter, The equation of state of solids from shock wave studies, in High-Velocity Impact Phenomena, edited by R. Kinslow, chapter 7, Academic Press, New York, New York, 1970.

9. S. J. Fillipov, N. B. Kazakov, and L. A. Pronin, Izv. Vyssh. Uchebn. Zaved. Chern. Metall. 9, 8 (1966).

10. R. G. McQueen, W. J. Carter, J. N. Fritz, and S. P. Marsh, The solid-liquid phase line in $\mathrm{Cu}$, in Accurate Characterization of the High-Pressure Environment, edited by E. C. Lloyd, pages 219-227, Washington, D. C., 1971, Nat. Bur. of Stds. Special Publication 326.

11. J. M. Brown and J. W. Shaner, Rarefaction velocities in shocked tantalum and the high-pressure melting point, in Shock Waves in Condensed Matter-1983, edited by J. R. Asay, R. A. Graham, and G. K. Straub, chapter II:17, pages 91-94, North Holland Physics Publishing, Amsterdam, 1984.

12. C. Yu, M. Suzuki, H. Shibata, and T. Emi, Materials Transactions JIM 37, 1251 (1996).

13. F. Inoko, T. Okada, and T. Yoshikawa, Defect and Diffusion Forum 156, 175 (1998).

14. G. G. III, S. Chen, and K. Vecchio, Metallurgical and Matls. Trans. 30A, 1235 (1999) 